\title{
Special Section Guest Editorial: Hyperspectral Remote Sensing and Imaging Spectrometer Design
}

\author{
Shen-En Qian, ${ }^{\text {a }}$ Robert O. Green, ${ }^{b}$ and Antonio J. Plaza ${ }^{c}$ \\ ${ }^{a}$ Canadian Space Agency, Saint-Hubert, Québec, Canada \\ bJet Propulsion Laboratory, California Institute of Technology, Pasadena, California, \\ United States \\ ${ }^{\mathrm{c}}$ University of Extremadura, Department of Technology of Computers \& Communication, \\ Cáceres, Spain
}

Hyperspectral imaging has been emerging as a new generation of remote sensing technology since the late 1980s when the first airborne hyperspectral instrument, AVIRIS, became operational followed by the launch of the first spaceborne hyperspectral sensor, Hyperion, onboard NASA's EO-1 satellite in 2000. The detailed spectral information provided by hyperspectral imagery and the spatial relationships in the neighborhood of pixels with different spectra often yield application results not possible with multispectral or other types of imagery.

Hyperspectral imaging technology goes well beyond traditional remote sensing applications ranging from agriculture, forestry, environmental monitoring, geology, defense and intelligence, and law enforcement to food safety and inspection and medical imaging. Research and development of processing techniques of hyperspectral imaging and hyperspectral applications is a hot area in the remote sensing community.

This special section aims to collect the most recent research and developments in data processing techniques and applications of hyperspectral remote sensing as well as hyperspectral imaging sensor design and implementation. Eight independent studies have been included in this special section after a vigorous peer review process. These studies cover: (1) atmospheric hyperspectral imaging for greenhouse gas and air quality, (2) radiative transfer modeling, (3) detection, classification and characterization, (4) data processing and exploitation methods and algorithms, (5) deep learning or machine learning based approaches for spectral processing, (6) pansharpening and super-resolution, and (7) de-noising.

Pandya et al. demonstrated the potential of airborne hyperspectral image data together with radiative transfer modeling to retrieve the atmospheric carbon dioxide concentration from point source emission of coal-fed super-thermal power plants. Chen et al. proposed a method for hyperspectral image classification using principal component analysis, 2D spatial convolution, and support vector machine and reported improved classification accuracy in comparison to existing methods. Cui et al. developed an iterative weighted classification method for hyperspectral images by combining active learning and transfer learning to avoid the use of a large number of labeled samples in conventional supervised classification methods. Li et al. estimated ship velocity based on wake characteristics retrieved from UAV-borne hyperspectral images. Zhang et al. presented work on unmixing hyperspectral images by employing super-pixels and low-rank double-sparse regression to improve the unmixing accuracy and lower the processing time. Pan et al. studied deep learning methods and developed a multi-stream deep ResCNN-based fusion method for pan-sharpening multispectral images. Better fusion results than state-ofthe-art technology were achieved. Guo, $\mathrm{Li}$, and $\mathrm{Li}$ reported a semisupervised self-learning method for lithological identification and mapping of spaceborne hyperspectral images to overcome the problem of insufficiently labeled training samples. Chen, Xie, and Qian utilized minimum noise fraction to separate a hyperspectral image into signal-related channels and noisy channels. The denoising process is applied only to the noisy channels using video block matching and 3D filtering.

These studies indicate that hyperspectral imaging is a powerful remote sensing technology. It allows qualitative and quantitative analyses in a broad range of remote sensing applications. The research and developments in data processing techniques and applications of hyperspectral

(C) 2021 Society of Photo-Optical Instrumentation Engineers (SPIE) 
images are very active in the remote sensing community. It was worth the effort to organize this special section to showcase the advancement of the research on this topic.

Finally, we would like to thank the authors of this special section for their contributions and offer great appreciations to the reviewers, the staff of the Journal of Applied Remote Sensing, and the SPIE Journals Department team for assistance with the high-quality peer review process of the manuscripts received. We hope that this special section will foster further research and development of hyperspectral imaging remote sensing. 